\title{
Runge-Kutta Type Methods for Directly Solving Special Fourth-Order Ordinary Differential Equations
}

\author{
Kasim Hussain, ${ }^{1,2}$ Fudziah Ismail, ${ }^{1,3}$ and Norazak Senu ${ }^{1,3}$ \\ ${ }^{1}$ Department of Mathematics, Faculty of Science, Universiti Putra Malaysia (UPM), 43400 Serdang, Selangor, Malaysia \\ ${ }^{2}$ Department of Mathematics, College of Science, Al-Mustansiriya University, Baghdad, Iraq \\ ${ }^{3}$ Institute for Mathematical Research, Universiti Putra Malaysia (UPM), 43400 Serdang, Selangor, Malaysia
}

Correspondence should be addressed to Fudziah Ismail; fudziah_i@yahoo.com.my

Received 25 May 2015; Revised 14 July 2015; Accepted 21 July 2015

Academic Editor: Yan-Jun Liu

Copyright (C) 2015 Kasim Hussain et al. This is an open access article distributed under the Creative Commons Attribution License, which permits unrestricted use, distribution, and reproduction in any medium, provided the original work is properly cited.

\begin{abstract}
A Runge-Kutta type method for directly solving special fourth-order ordinary differential equations (ODEs) which is denoted by RKFD method is constructed. The order conditions of RKFD method up to order five are derived; based on the order conditions, three-stage fourth- and fifth-order Runge-Kutta type methods are constructed. Zero-stability of the RKFD method is proven. Numerical results obtained are compared with the existing Runge-Kutta methods in the scientific literature after reducing the problems into a system of first-order ODEs and solving them. Numerical results are presented to illustrate the robustness and competency of the new methods in terms of accuracy and number of function evaluations.
\end{abstract}

\section{Introduction}

This paper deals with the numerical integration of the special fourth-order ordinary differential equations (ODEs) of the form

$$
y^{(i v)}(x)=f(x, y),
$$

with initial conditions

$$
\begin{gathered}
y\left(x_{0}\right)=y_{0}, \\
y^{\prime}\left(x_{0}\right)=y_{0}^{\prime}, \\
y^{\prime \prime}\left(x_{0}\right)=y_{0}^{\prime \prime}, \\
y^{\prime \prime \prime}\left(x_{0}\right)=y_{0}^{\prime \prime \prime},
\end{gathered}
$$

in which the first, second, and third derivatives do not appear explicitly. This type of problems often arises in many fields of applied science such as mechanics, astrophysics, quantum chemistry, and electronic and control engineering. The general approach for solving the higher-order ordinary differential equation (ODE) is by transforming it into an equivalent first-order system of differential equations and then applying the appropriate numerical methods to solve the resulting system (see [1-5]). However, the application of such technique takes a lot of computational time (see $[6,7])$. Direct integration method is proposed to avoid such computational encumbrance and increase the efficiency of the method. Many authors have proposed several numerical methods for directly approximating the solutions for the higher-order ODEs; for example, Kayode [8] proposed zero-stable predictor-corrector methods for solving fourthorder ordinary differential equations. Majid and Suleiman [9] derived one point method to solve system of higherorder ODEs. Jain et al. [10] constructed finite difference method for solving fourth-order ODEs. Waeleh et al. [11] constructed a new block method for solving directly higherorder ODEs. Awoyemi and Idowu [12] proposed a hybrid collocations method for solving third-order ODEs. Hybrid linear multistep method with three steps to solve secondorder ODEs was introduced by Jator [13], and all the methods discussed above are multistep in nature.

This paper primarily aims to construct a one-step method of orders four and five to solve special fourth-order ODEs directly; these new methods are self-starting in nature. The paper is organized as follows. In Section 2, the derivation 
of the order conditions of RKFD method is presented. In Section 3, the zero-stability of RKFD method is given. In Section 4, three-stage RKFD methods of order four and order five are constructed. In Section 5, numerical examples are given to show the effectiveness and competency of the new RKFD methods as compared with the well known RungeKutta methods from the scientific literature. Conclusions are given in Section 6.

\section{Derivation of the RKFD Method}

The general form of RKFD method with $s$-stage for directly solving special fourth-order ODEs (1) can be written as follows:

$$
\begin{aligned}
& y_{n+1}=y_{n}+h y_{n}^{\prime}+\frac{h^{2}}{2} y_{n}^{\prime \prime}+\frac{h^{3}}{6} y_{n}^{\prime \prime \prime}+h^{4} \sum_{i=1}^{s} b_{i} k_{i}, \\
& y_{n+1}^{\prime}=y_{n}^{\prime}+h^{2} y_{n}^{\prime \prime}+\frac{h^{2}}{2} y_{n}^{\prime \prime \prime}+h^{3} \sum_{i=1}^{s} b_{i}^{\prime} k_{i}, \\
& y_{n+1}^{\prime \prime}=y_{n}^{\prime \prime}+h y_{n}^{\prime \prime \prime}+h^{2} \sum_{i=1}^{s} b_{i}^{\prime \prime} k_{i}, \\
& y_{n+1}^{\prime \prime \prime}=y_{n}^{\prime \prime \prime}+h \sum_{i=1}^{s} b_{i}^{\prime \prime \prime} k_{i},
\end{aligned}
$$

where

$$
\begin{aligned}
k_{1} & =f\left(x_{n}, y_{n}\right), \\
k_{i} & =f\left(x_{n}+c_{i} h, y_{n}+h c_{i} y_{n}^{\prime}+\frac{h^{2}}{2} c_{i}^{2} y_{n}^{\prime \prime}+\frac{h^{3}}{6} c_{i}^{3} y_{n}^{\prime \prime \prime}\right. \\
& \left.+h^{4} \sum_{j=1}^{i-1} a_{i j} k_{j}\right) . \quad i=2,3, \ldots, s .
\end{aligned}
$$

All parameters $b_{i}, b_{i}^{\prime}, b_{i}^{\prime \prime}, b_{i}^{\prime \prime \prime}, a_{i j}$, and $c_{i}$ of the RKFD method are used for $i, j=1,2, \ldots, s$ and are supposed to be real. The RKFD method is an explicit method if $a_{i j}=0$ for $i \leq j$ and is an implicit method if $a_{i j} \neq 0$ for $i \leq j$. The RKFD method can be represented by Butcher tableau as follows:

$$
\begin{array}{c|c}
c & A \\
\hline & b^{T} \\
b^{\prime T} \\
b^{\prime \prime T} \\
b^{\prime \prime \prime T}
\end{array}
$$

To determine the parameters of the RKFD method given in (3)-(8), the RKFD method expression is expanded using the Taylor series expansion. After performing some algebraic manipulations, this expansion is equated to the true solution that is given by the Taylor series expansion. The direct expansion of the local truncation error is used to derive the general order conditions for the RKFD method. This approach depends on the derivation of order conditions for the Runge-Kutta method proposed in Dormand [14]. The RKFD method in (3)-(6) can be written as follows:

$$
\begin{aligned}
& y_{n+1}=y_{n}+h \psi\left(x_{n}, y_{n}\right), \\
& y_{n+1}^{\prime}=y_{n}^{\prime}+h \psi^{\prime}\left(x_{n}, y_{n}\right), \\
& y_{n+1}^{\prime \prime}=y_{n}^{\prime \prime}+h \psi^{\prime \prime}\left(x_{n}, y_{n}\right), \\
& y_{n+1}^{\prime \prime \prime}=y_{n}^{\prime \prime \prime}+h \psi^{\prime \prime \prime}\left(x_{n}, y_{n}\right),
\end{aligned}
$$

where the increment functions are

$$
\begin{aligned}
\psi\left(x_{n}, y_{n}\right) & =y_{n}^{\prime}+\frac{h}{2} y_{n}^{\prime \prime}+\frac{h^{2}}{6} y_{n}^{\prime \prime \prime}+h^{3} \sum_{i=1}^{s} b_{i} k_{i}, \\
\psi^{\prime}\left(x_{n}, y_{n}\right) & =y_{n}^{\prime \prime}+\frac{h}{2} y_{n}^{\prime \prime \prime}+h^{2} \sum_{i=1}^{s} b_{i}^{\prime} k_{i}, \\
\psi^{\prime \prime}\left(x_{n}, y_{n}\right) & =y_{n}^{\prime \prime \prime}+h \sum_{i=1}^{s} b_{i}^{\prime \prime} k_{i}, \\
\psi^{\prime \prime \prime}\left(x_{n}, y_{n}\right) & =\sum_{i=1}^{s} b_{i}^{\prime \prime \prime} k_{i},
\end{aligned}
$$

where $k_{i}$ is given in (8).

The first few elementary differentials for the scalar equation are

$$
\begin{aligned}
F_{1}^{(4)}= & y^{(i v)}=f, \\
F_{1}^{(5)}= & f_{x}+f_{y} y^{\prime}, \\
F_{1}^{(6)}= & f_{x x}+2 f_{x y} y^{\prime}+f_{y} y^{\prime \prime}+f_{y y}\left(y^{\prime}\right)^{2}, \\
F_{1}^{(7)}= & f_{x x x}+3 f_{x x y} y^{\prime}+3 f_{x y y}\left(y^{\prime}\right)^{2}+3 f_{x y} y^{\prime \prime} \\
& +3 f_{y y} y^{\prime} y^{\prime \prime}+f_{y y y}\left(y^{\prime}\right)^{3}+f_{y} y^{\prime \prime \prime} .
\end{aligned}
$$

We assume that the Taylor series increment function is $\Delta$.

The local truncation errors of $y(x), y^{\prime}(x), y^{\prime \prime}(x)$, and $y^{\prime \prime \prime}(x)$ can be obtained after substituting the exact solution of (1) into (11) as follows:

$$
\begin{aligned}
\tau_{n+1} & =h[\psi-\Delta], \\
\tau_{n+1}^{\prime} & =h\left[\psi^{\prime}-\Delta^{\prime}\right], \\
\tau_{n+1}^{\prime \prime} & =h\left[\psi^{\prime \prime}-\Delta^{\prime \prime}\right], \\
\tau_{n+1}^{\prime \prime \prime} & =h\left[\psi^{\prime \prime \prime}-\Delta^{\prime \prime \prime}\right] .
\end{aligned}
$$


The Taylor series increment functions of $y(x), y^{\prime}(x), y^{\prime \prime}(x)$, and $y^{\prime \prime \prime}(x)$ can be expressed as follows:

$$
\begin{aligned}
\Delta= & y^{\prime}+\frac{1}{2} h y^{\prime \prime}+\frac{1}{6} h^{2} y^{\prime \prime \prime}+\frac{1}{24} h^{3} F_{1}^{(4)}+\frac{1}{120} h^{4} F_{1}^{(5)} \\
& +\frac{1}{720} h^{5} F_{1}^{(6)}+O\left(h^{6}\right), \\
\Delta^{\prime}= & y^{\prime \prime}+\frac{1}{2} h y^{\prime \prime \prime}+\frac{1}{6} h^{2} F_{1}^{(4)}+\frac{1}{24} h^{3} F_{1}^{(5)} \\
& +\frac{1}{120} h^{4} F_{1}^{(6)}+\frac{1}{720} h^{5} F_{1}^{(7)}+O\left(h^{6}\right), \\
\Delta^{\prime \prime}= & y^{\prime \prime \prime}+\frac{1}{2} h F_{1}^{(4)}+\frac{1}{6} h^{2} F_{1}^{(5)}+\frac{1}{24} h^{3} F_{1}^{(6)} \\
& +\frac{1}{120} h^{4} F_{1}^{(7)}+\frac{1}{720} h^{5} F_{1}^{(8)}+O\left(h^{6}\right), \\
\Delta^{\prime \prime \prime}= & F_{1}^{(4)}+\frac{1}{2} h F_{1}^{(5)}+\frac{1}{6} h^{2} F_{1}^{(6)}+\frac{1}{24} h^{3} F_{1}^{(7)} \\
& +\frac{1}{720} h^{4} F_{1}^{(8)}+O\left(h^{5}\right) .
\end{aligned}
$$

Substituting (12) into (11), the increment functions $\psi, \psi^{\prime}, \psi^{\prime \prime}$, and $\psi^{\prime \prime \prime}$ for RKFD method become as follows:

$$
\begin{aligned}
& \sum_{i=1}^{s} b_{i} k_{i} \\
& =\sum_{i=1}^{s} b_{i} f+\sum_{i=1}^{s} b_{i} c_{i}\left(f_{x}+f_{y} y^{\prime}\right) h \\
& \quad+\frac{1}{2} \sum_{i=1}^{s} b_{i} c_{i}^{2}\left(f_{x x}+2 f_{x y} y^{\prime}+f_{y} y^{\prime \prime}+f_{y y}\left(y^{\prime}\right)^{2}\right) h^{2} \\
& \quad+O\left(h^{3}\right), \\
& \sum_{i=1}^{s} b_{i} k_{i} \\
& =\sum_{i=1}^{s} b_{i} F_{1}^{(4)}+\sum_{i=1}^{s} b_{i} c_{i} h F_{1}^{(5)}+\frac{1}{2} \sum_{i=1}^{s} b_{i} c_{i}^{2} h^{2} F_{1}^{(6)}+O\left(h^{3}\right),
\end{aligned}
$$$$
\sum_{i=1}^{s} b_{i}^{\prime} k_{i}
$$$$
=\sum_{i=1}^{s} b_{i}^{\prime} f+\sum_{i=1}^{s} b_{i}^{\prime} c_{i}\left(f_{x}+f_{y} y^{\prime}\right) h
$$$$
+\frac{1}{2} \sum_{i=1}^{s} b_{i}^{\prime} c_{i}^{2}\left(f_{x x}+2 f_{x y} y^{\prime}+f_{y} y^{\prime \prime}+f_{y y}\left(y^{\prime}\right)^{2}\right) h^{2}
$$$$
+O\left(h^{3}\right) \text {, }
$$

$\sum_{i=1}^{s} b_{i}^{\prime} k_{i}$

$$
=\sum_{i=1}^{s} b_{i}^{\prime} F_{1}^{(4)}+\sum_{i=1}^{s} b_{i}^{\prime} c_{i} h F_{1}^{(5)}+\frac{1}{2} \sum_{i=1}^{s} b_{i}^{\prime} c_{i}^{2} h^{2} F_{1}^{(6)}+O\left(h^{3}\right),
$$

$\sum_{i=1}^{s} b_{i}^{\prime \prime} k_{i}$

$$
\begin{aligned}
= & \sum_{i=1}^{s} b_{i}^{\prime \prime} f+\sum_{i=1}^{s} b_{i}^{\prime \prime} c_{i}\left(f_{x}+f_{y} y^{\prime}\right) h \\
& +\frac{1}{2} \sum_{i=1}^{s} b_{i}^{\prime \prime} c_{i}^{2}\left(f_{x x}+2 f_{x y} y^{\prime}+f_{y} y^{\prime \prime}+f_{y y}\left(y^{\prime}\right)^{2}\right) h^{2} \\
& +O\left(h^{3}\right),
\end{aligned}
$$

$\sum_{i=1}^{s} b_{i}^{\prime \prime} k_{i}$

$=\sum_{i=1}^{s} b_{i}^{\prime \prime} F_{1}^{(4)}+\sum_{i=1}^{s} b_{i}^{\prime \prime} c_{i} h F_{1}^{(5)}+\frac{1}{2} \sum_{i=1}^{s} b_{i}^{\prime \prime} c_{i}^{2} h^{2} F_{1}^{(6)}$

$+O\left(h^{3}\right)$,

$\sum_{i=1}^{s} b_{i}^{\prime \prime \prime} k_{i}$

$$
\begin{aligned}
& =\sum_{i=1}^{s} b_{i}^{\prime \prime \prime} f+\sum_{i=1}^{s} b_{i}^{\prime \prime \prime} c_{i}\left(f_{x}+f_{y} y^{\prime}\right) h \\
& \quad+\frac{1}{2} \sum_{i=1}^{s} b_{i}^{\prime \prime \prime} c_{i}^{2}\left(f_{x x}+2 f_{x y} y^{\prime}+f_{y} y^{\prime \prime}+f_{y y}\left(y^{\prime}\right)^{2}\right) h^{2} \\
& \quad+O\left(h^{3}\right), \\
& \sum_{i=1}^{s} b_{i}^{\prime \prime \prime} k_{i} \\
& =\sum_{i=1}^{s} b_{i}^{\prime \prime \prime} F_{1}^{(4)}+\sum_{i=1}^{s} b_{i}^{\prime \prime \prime} c_{i} h F_{1}^{(5)}+\frac{1}{2} \sum_{i=1}^{s} b_{i}^{\prime \prime \prime} c_{i}^{2} h^{2} F_{1}^{(6)} \\
& \quad+O\left(h^{3}\right) .
\end{aligned}
$$

Using (11) and (14), the local truncation errors (13) can be written as follows:

$$
\begin{aligned}
\tau_{n+1} & =h^{4}\left[\sum_{i=1}^{s} b_{i} k_{i}-\left(\frac{1}{24} F_{1}^{(4)}+\frac{1}{120} h F_{1}^{(5)}+\cdots\right)\right], \\
\tau_{n+1}^{\prime} & =h^{3}\left[\sum_{i=1}^{s} b_{i}^{\prime} k_{i}-\left(\frac{1}{6} F_{1}^{(4)}+\frac{1}{24} h F_{1}^{(5)}+\cdots\right)\right], \\
\tau_{n+1}^{\prime \prime} & =h^{2}\left[\sum_{i=1}^{s} b_{i}^{\prime \prime} k_{i}-\left(\frac{1}{2} F_{1}^{(4)}+\frac{1}{6} h F_{1}^{(5)}+\cdots\right)\right], \\
\tau_{n+1}^{\prime \prime \prime} & \\
= & h\left[\sum_{i=1}^{s} b_{i}^{\prime \prime \prime} k_{i}-\left(F_{1}^{(4)}+\frac{1}{2} h F_{1}^{(5)}+\frac{1}{6} h^{2} F_{1}^{(6)}+\cdots\right)\right] .
\end{aligned}
$$


By offsetting (15) into (16) and expanding as a Taylor series expansion using computer algebra package MAPLE (see [15]), the local truncation errors or the order conditions for $s$ stage fifth-order RKFD method can be written as follows.

The order conditions for $y$ are

fourth order:

$$
\sum_{i=1}^{s} b_{i}=\frac{1}{24}
$$

fifth order:

$$
\sum_{i=1}^{s} b_{i} c_{i}=\frac{1}{120} .
$$

The order conditions for $y^{\prime}$ are

third order:

$$
\sum_{i=1}^{s} b_{i}^{\prime}=\frac{1}{6}
$$

fourth order:

$$
\sum_{i=1}^{s} b_{i}^{\prime} c_{i}=\frac{1}{24}
$$

fifth order:

$$
\sum_{i=1}^{s} b_{i}^{\prime} c_{i}^{2}=\frac{1}{60}
$$

The order conditions for $y^{\prime \prime}$ are

second order:

$$
\sum_{i=1}^{s} b_{i}^{\prime \prime}=\frac{1}{2}
$$

third order:

$$
\sum_{i=1}^{s} b_{i}^{\prime \prime} c_{i}=\frac{1}{6}
$$

fourth order:

$$
\sum_{i=1}^{s} b_{i}^{\prime \prime} c_{i}^{2}=\frac{1}{12}
$$

fifth order:

$$
\sum_{i=1}^{s} b_{i}^{\prime \prime} c_{i}^{3}=\frac{1}{20} .
$$

The order conditions for $y^{\prime \prime \prime}$ are

first order:

$$
\sum_{i=1}^{s} b_{i}^{\prime \prime \prime}=1
$$

second order:

$$
\sum_{i=1}^{s} b_{i}^{\prime \prime \prime} c_{i}=\frac{1}{2},
$$

third order:

$$
\sum_{i=1}^{s} b_{i}^{\prime \prime \prime} c_{i}^{2}=\frac{1}{3}
$$

fourth order:

$$
\sum_{i=1}^{s} b_{i}^{\prime \prime \prime} c_{i}^{3}=\frac{1}{4}
$$

fifth order:

$$
\begin{gathered}
\sum_{i=1}^{s} b_{i}^{\prime \prime \prime} c_{i}^{4}=\frac{1}{5} \\
\sum_{i, j=1}^{s} b_{i}^{\prime \prime \prime} a_{i j}=\frac{1}{120} .
\end{gathered}
$$

\section{Zero-Stability of the RKFD Method}

In this section, we discuss the convergence of the RKFD method by introducing the concept of zero-stability of the RKFD method. A good numerical method is a method in which the numerical approximation to the solution converges, and zero-stability is a significant criterion for convergence. The zero-stability concept for those numerical methods that are used for solving first- and second-order ODEs can be seen in Lambert [16], Dormand [14], and Butcher [4]. The RKFD method (3)-(8) can be expressed in the matrix form as follows:

$$
\left[\begin{array}{llll}
1 & 0 & 0 & 0 \\
0 & 1 & 0 & 0 \\
0 & 0 & 1 & 0 \\
0 & 0 & 0 & 0
\end{array}\right]\left[\begin{array}{c}
y_{n+1} \\
h y_{n+1}^{\prime} \\
h^{2} y_{n+1}^{\prime \prime} \\
h^{3} y_{n+1}^{\prime \prime \prime}
\end{array}\right]=\left[\begin{array}{llll}
1 & 1 & \frac{1}{2} & \frac{1}{6} \\
0 & 1 & 1 & \frac{1}{2} \\
0 & 0 & 1 & 1 \\
0 & 0 & 0 & 1
\end{array}\right]\left[\begin{array}{c}
y_{n} \\
h y_{n}^{\prime} \\
h^{2} y_{n}^{\prime \prime} \\
h^{3} y_{n}^{\prime \prime \prime}
\end{array}\right],
$$

where $I=\left[\begin{array}{llll}1 & 0 & 0 & 0 \\ 0 & 1 & 0 & 0 \\ 0 & 0 & 1 & 0 \\ 0 & 0 & 0 & 0\end{array}\right]$ is the identity matrix coefficients of $y_{n+1}$, $h y_{n+1}^{\prime}, h^{2} y_{n+1}^{\prime \prime}$, and $h^{3} y_{n+1}^{\prime \prime \prime}$, respectively, and $A=\left[\begin{array}{cccc}1 & 1 & 1 / 2 & 1 / 6 \\ 0 & 1 & 1 & 1 / 2 \\ 0 & 0 & 1 \\ 0 & 0 & 0 & 1\end{array}\right]$ is matrix coefficients of $y_{n}, h y_{n}^{\prime}, h^{2} y_{n}^{\prime \prime}$, and $h^{3} y_{n}^{\prime \prime \prime}$, respectively.

The characteristic polynomial of the RKFD method is denoted by $\varrho(\zeta)$ which can be written as follows:

$$
\varrho(\zeta)=|I \zeta-A|=\left|\begin{array}{cccc}
\zeta-1 & -1 & -\frac{1}{2} & -\frac{1}{6} \\
0 & \zeta-1 & -1 & -\frac{1}{2} \\
0 & 0 & \zeta-1 & -1 \\
0 & 0 & 0 & \zeta-1
\end{array}\right| .
$$

Hence, $\varrho(\zeta)=(\zeta-1)^{4}$.

We find that all the roots are $\zeta=1,1,1,1$. Generalizing the theorem proposed by Henrici [17] for solving special second-order ODEs, therefore, the RKFD method is zerostable since all roots are less than or equal to the value of 1 . 


\section{Construction of RKFD Methods}

In this section, we proceed to construct explicit RKFD methods based on the order conditions which we have derived in Section 2.

4.1. A Three-Stage RKFD Method of Order Four. This section will focus on the derivation of a three-stage RKFD method of order four, where we use the algebraic order conditions (17), (19)-(20), (22)-(24), and (26)-(29), respectively. The resulting system of equations consists of 10 nonlinear equations with 14 unknown variables to be solved; solving the system simultaneously yields a solution with four free parameters $c_{3}$, $b_{1}^{\prime}, b_{1}$, and $b_{3}$ as follows:

$$
\begin{aligned}
c_{2} & =-\frac{3-4 c_{3}}{-4+6 c_{3}}, \\
b_{1}^{\prime \prime \prime} & =\frac{6 c_{3}^{2}-6 c_{3}+1}{6 c_{3}\left(-3+4 c_{3}\right)}, \\
b_{2}^{\prime \prime \prime} & =-\frac{16-72 c_{3}+108 c_{3}^{2}-54 c_{3}^{3}}{108 c_{3}-150 c_{3}^{2}-27+72 c_{3}^{3}}, \\
b_{3}^{\prime \prime \prime} & =\frac{1}{36 c_{3}^{3}-48 c_{3}^{2}+18 c_{3}}, \\
b_{1}^{\prime \prime} & =\frac{6 c_{3}^{2}-6 c_{3}+1}{6 c_{3}\left(-3+4 c_{3}\right)}, \\
b_{2}^{\prime \prime} & =-\frac{-20 c_{3}-18 c_{3}^{3}+33 c_{3}^{2}+4}{108 c_{3}-150 c_{3}^{2}-27+72 c_{3}^{3}}, \\
b_{3}^{\prime \prime} & =-\frac{-1+c_{3}}{36 c_{3}^{3}-48 c_{3}^{2}+18 c_{3}}, \\
b_{2}^{\prime} & =-\frac{-48 b_{1}^{\prime} c_{3}+72 b_{1}^{\prime} c_{3}^{2}-2+11 c_{3}-12 c_{3}^{2}}{12\left(3-8 c_{3}+6 c_{3}^{2}\right)},
\end{aligned}
$$

$$
\begin{aligned}
& b_{3}^{\prime}=-\frac{-4+36 b_{1}^{\prime}+5 c_{3}-48 b_{1}^{\prime} c_{3}}{-96 c_{3}+72 c_{3}^{2}+36}, \\
& b_{2}=-b_{1}-b_{3}+\frac{1}{24} .
\end{aligned}
$$

Thus, these free parameters can be chosen by minimizing the local truncation error norms of the fifth-order conditions according to Dormand et al. [18]. However, we have another three free parameters $a_{21}, a_{31}$, and $a_{32}$ that do not appear in fourth-order conditions but they appear in the minimization of error equations for fifth-order conditions of $y^{\prime \prime \prime}$.

The error norms and global error of fifth-order conditions are defined as follows:

$$
\begin{aligned}
& \left\|\tau^{(5)}\right\|_{2}=\sqrt{\sum_{i=1}^{n_{p}+1}\left(\tau_{i}^{(5)}\right)^{2},} \\
& \left\|\tau^{\prime(5)}\right\|_{2}=\sqrt{\sum_{i=1}^{n_{p}^{\prime}+1}\left(\tau_{i}^{\prime(5)}\right)^{2},} \\
& \left\|\tau^{\prime \prime(5)}\right\|_{2}=\sqrt{\sum_{i=1}^{n_{p}^{\prime \prime}+1}\left(\tau_{i}^{\prime \prime(5)}\right)^{2},} \\
& \left\|\tau^{\prime \prime \prime(5)}\right\|_{2}=\sqrt{\sum_{i=1}^{n_{p}^{\prime \prime \prime}+1}\left(\tau_{i}^{\prime \prime \prime}(5)\right)^{2}}, \\
& \left\|\tau_{g}^{(5)}\right\|_{2} \\
& =\sqrt{\sum_{i=1}^{n_{p}+1}\left(\tau_{i}^{(5)}\right)^{2}+\sum_{i=1}^{n_{p}^{\prime}+1}\left(\tau_{i}^{\prime(5)}\right)^{2}+\sum_{i=1}^{n_{p}^{\prime \prime}+1}\left(\tau_{i}^{\prime \prime(5)}\right)^{2}+\sum_{i=1}^{n_{p}^{\prime \prime \prime}+1}\left(\tau_{i}^{\prime \prime \prime(5)}\right)^{2}},
\end{aligned}
$$

where $\tau^{(5)}, \tau^{\prime(5)}, \tau^{\prime \prime(5)}$, and $\tau^{\prime \prime \prime(5)}$ are the local truncation error norms for $y, y^{\prime}, y^{\prime \prime}$, and $y^{\prime \prime \prime}$, respectively, and $\tau_{g}^{(5)}$ is the global error.

Consequently, we find the error norms of $y, y^{\prime}$, and $y^{\prime \prime}$, respectively, as follows:

$$
\begin{aligned}
& \left\|\tau^{(5)}\right\|_{2}=\frac{1}{240} \sqrt{\frac{\left(-360 b_{3}+11-720 c_{3}^{2} b_{3}+480 b_{1} c_{3}-360 b_{1}+960 b_{3} c_{3}-14 c_{3}\right)^{2}}{\left(-2+3 c_{3}\right)^{2}}}, \\
& \left\|\tau^{\prime(5)}\right\|_{2}=\frac{1}{240} \sqrt{\frac{\left(-50 c_{3}^{2}+480 b_{1}^{\prime} c_{3}^{2}-360 b_{1}^{\prime} c_{3}+48 c_{3}-7\right)^{2}}{\left(-2+3 c_{3}\right)^{2}}}, \\
& \left\|\tau^{\prime \prime(5)}\right\|_{2}=\frac{1}{120} \sqrt{\frac{\left(3-12 c_{3}+10 c_{3}^{2}\right)^{2}}{\left(-2+3 c_{3}\right)^{2}}} .
\end{aligned}
$$


Our goal is to choose the free parameters $c_{3}, b_{1}^{\prime}, b_{1}$, and $b_{3}$ such that the error norms of fifth-order conditions have minimal value. By plotting the graph of $\left\|\tau^{\prime \prime(5)}\right\|_{2}$ versus $c_{3}$ and choosing a small value of $c_{3}$ in the interval $[0.7,3]$, we find that $c_{3}=$ $17 / 20$ is the optimal value which yields a minimum value for $\left\|\tau^{\prime \prime(5)}\right\|_{2}=3.787878788 \times 10^{-4}$. Substituting the value of $c_{3}=$ $17 / 20$ into $\left\|\tau^{(5)}\right\|_{2}$ and $\left\|\tau^{\prime(5)}\right\|_{2}$ we get

$$
\begin{aligned}
\left\|\tau^{(5)}\right\|_{2} & =\frac{1}{440} \sqrt{\left(3-160 b_{1}+214 b_{3}\right)^{2}}, \\
\left\|\tau^{\prime(5)}\right\|_{2} & =\frac{1}{1760} \sqrt{\left(-31+544 b_{1}^{\prime}\right)^{2}} .
\end{aligned}
$$

Also through plotting the graph of $\left\|\tau^{(5)}\right\|_{2}$ against $b_{1}$ and $b_{3}$ in the interval $[-0.1,0.5]$ and choosing a small value of $b_{3}$, we get that $b_{3}=1 / 20$ is the optimal value which gives $b_{1}=$ $17 / 200$ and $\left\|\tau^{(5)}\right\|_{2}=2.272727273 \times 10^{-4}$.

Now, utilizing the same technique where we draw the graph of $\left\|\tau^{\prime(5)}\right\|_{2}$ versus $b_{1}^{\prime}$ in the interval $[-1,1]$, we find that $b_{1}^{\prime}=1 / 18$ is the best choice, and with this value of $b_{1}^{\prime}$, we get $\left\|\tau^{\prime(5)}\right\|_{2}=4.419191919 \times 10^{-4}$.

Therefore, the error equation of the fifth-order condition of $y^{\prime \prime \prime}$ is as follows:

$$
\begin{aligned}
& \left\|\tau^{\prime \prime \prime(5)}\right\|_{2}=\frac{1}{4802160}(1604749085 \\
& \quad+6194971660900 a_{21}^{2}+8761174400 a_{21} a_{31} \\
& \quad+8761174400 a_{21} a_{32}-199207202920 a_{21} \\
& \quad+3097600 a_{31}^{2}+6195200 a_{31} a_{32}-1408633600 a_{31} \\
& \left.+3097600 a_{32}^{2}-1408633600 a_{32}\right)^{1 / 2} .
\end{aligned}
$$

Consequently, the global error is

$$
\begin{aligned}
& \left\|\tau_{g}^{(5)}\right\|_{2}=\frac{1}{144064800}(1452377332189 \\
& +55754744948100 a_{21}^{2}+78850569600 a_{21} a_{31} \\
& +78850569600 a_{21} a_{32}-17928648262800 a_{21} \\
& +27878400 a_{31}^{2}+55756800 a_{31} a_{32} \\
& -12677702400 a_{31}+27878400 a_{32}^{2} \\
& \left.-12677702400 a_{32}\right)^{1 / 2} .
\end{aligned}
$$

By minimizing the error norm in (37) and global error in (38) with respect to the free parameters $a_{21}, a_{31}$, and $a_{32}$, we get $a_{21}=-1 / 5, a_{31}=19 / 125$, and $a_{32}=19 / 125$, which produces $\left\|\tau^{\prime \prime \prime(5)}\right\|_{2}=3.7878787879 \times 10^{-4}$ and $\left\|\tau_{g}^{(5)}\right\|_{2}=7.3068870183 \times$ $10^{-4}$. Finally, all the coefficients of three-stage fourth-order
RKFD method are written in Butcher tableau and denoted by RKFD4 method as follows:

$$
\begin{array}{c|ccc}
\frac{4}{11} & -\frac{1}{5} & & \\
\frac{17}{20} & \frac{19}{125} & \frac{19}{125} & \\
\hline & \frac{17}{200} & -\frac{7}{75} & \frac{1}{20} \\
\frac{1}{18} & \frac{209}{1926} & \frac{5}{1926} \\
\frac{47}{408} & \frac{847}{2568} & \frac{100}{1819} \\
\frac{47}{408} & \frac{1331}{2568} & \frac{2000}{5457}
\end{array}
$$

4.2. A Three-Stage RKFD Method of Order Five. In this section, a three-stage RKFD method of order five will be derived. The algebraic order conditions up to order five ((17)(18), (19)-(21), (22)-(25), and (26)-(30)) need to be solved. The resulting system of equations consists of fifteen nonlinear equations, solving the system simultaneously which results in a solution with three free parameters $b_{1}, a_{21}$, and $a_{31}$ as follows:

$$
\begin{aligned}
& c_{2}=\frac{3}{5}+\frac{\sqrt{6}}{10}, \\
& c_{3}=\frac{3}{5}-\frac{\sqrt{6}}{10}, \\
& b_{1}^{\prime \prime \prime}=\frac{1}{9}, \\
& b_{2}^{\prime \prime \prime}=\frac{4}{9}-\frac{\sqrt{6}}{36}, \\
& b_{3}^{\prime \prime \prime}=\frac{4}{9}+\frac{\sqrt{6}}{36}, \\
& b_{1}^{\prime \prime}=\frac{1}{9}, \\
& b_{2}^{\prime \prime}=\frac{7}{36}-\frac{\sqrt{6}}{18}, \\
& b_{3}^{\prime \prime}=\frac{1}{18}+\frac{7}{48}, \frac{\sqrt{6}}{18}, \\
& b_{2}^{\prime}=\frac{1}{18}-\frac{\sqrt{6}}{48},
\end{aligned}
$$




$$
\begin{aligned}
& b_{2}=\frac{1}{48}-\frac{\sqrt{6}}{72}+\left(-\frac{1}{2}+\frac{\sqrt{6}}{2}\right) b_{1}, \\
& b_{3}=\frac{1}{48}+\frac{\sqrt{6}}{72}-\left(\frac{1}{2}+\frac{\sqrt{6}}{2}\right) b_{1}, \\
& a_{32}=\left(-\frac{131}{125}+\frac{16 \sqrt{6}}{125}\right) a_{21}-a_{31}+\frac{12}{625}-\frac{3 \sqrt{6}}{2500} .
\end{aligned}
$$

Thus, these free parameters can be chosen by minimizing the local truncation error norms of the sixth-order conditions. The error norms and the global error of the sixth-order conditions are given by

$$
\begin{aligned}
& \left\|\tau^{(6)}\right\|_{2}=\sqrt{\sum_{i=1}^{n_{p}+1}\left(\tau_{i}^{(6)}\right)^{2}}, \\
& \left\|\tau^{\prime(6)}\right\|_{2}=\sqrt{\sum_{i=1}^{n_{p}^{\prime}+1}\left(\tau_{i}^{\prime(6)}\right)^{2}} \\
& \left\|\tau^{\prime^{\prime \prime(6)}}\right\|_{2}=\sqrt{\sum_{i=1}^{n_{p}^{\prime \prime+1}}\left(\tau_{i}^{\prime \prime(6)}\right)^{2}} \\
& \left\|\tau^{\prime \prime \prime(6)}\right\|_{2}=\sqrt{\sum_{i=1}^{n_{p}^{\prime \prime \prime}+1}\left(\tau_{i}^{\prime \prime \prime(6)}\right)^{2}} \\
& \left\|\tau_{g}^{(6)}\right\|_{2} \\
& =\sqrt{\sum_{i=1}^{n_{p}+1}\left(\tau_{i}^{(6)}\right)^{2}+\sum_{i=1}^{n_{p}^{\prime}+1}\left(\tau_{i}^{\prime(6)}\right)^{2}+\sum_{i=1}^{n_{p}^{\prime \prime}+1}\left(\tau_{i}^{\prime \prime(6)}\right)^{2}+\sum_{i=1}^{n_{p}^{\prime \prime \prime}+1}\left(\tau_{i}^{\prime \prime \prime(6)}\right)^{2}},
\end{aligned}
$$

where $\tau^{(6)}, \tau^{\prime(6)}, \tau^{\prime \prime(6)}$, and $\tau^{\prime \prime \prime(6)}$ are the local truncation error norms for $y, y^{\prime}, y^{\prime \prime}$, and $y^{\prime \prime \prime}$ of the RKFD method, respectively. $\tau_{g}^{(6)}$ is the global error. The error equation of sixth-order condition for $y$ with respect to the free parameter $b_{1}$ is as follows:

$$
\left\|\tau^{(6)}\right\|_{2}=\frac{1}{3600} \sqrt{\left(-19+1080 b_{1}\right)^{2}} .
$$

The error equation $\left\|\tau^{(6)}\right\|_{2}$ has a minimum value equal to zero at $b_{1}=19 / 1080 \approx 0.01759259259$ which leads to $b_{2}=$ $13 / 1080-11 \sqrt{6} / 2160$ and $b_{3}=13 / 1080+11 \sqrt{6} / 2160$. The truncation error norms of the sixth-order condition of $y, y^{\prime}$, $y^{\prime \prime}$, and $y^{\prime \prime \prime}$ are calculated as follows:

$$
\begin{aligned}
& \left\|\tau^{(6)}\right\|_{2}=0 \\
& \left\|\tau^{\prime(6)}\right\|_{2}=\frac{1}{1200}, \\
& \left\|\tau^{\prime \prime(6)}\right\|_{2}=\frac{1}{3600}\left(139+628800 a_{21}^{2}-76800 a_{21}^{2} \sqrt{6}\right. \\
& \left.\quad-9840 a_{21}-3760 a_{21} \sqrt{6}+42 \sqrt{6}\right)^{1 / 2},
\end{aligned}
$$

$$
\begin{aligned}
& \left\|\tau^{\prime \prime \prime(6)}\right\|_{2}=\frac{1}{3600}\left(362-11760 a_{21} \sqrt{6}+103200 a_{21}^{2} \sqrt{6}\right. \\
& +600000 a_{21} \sqrt{6} a_{31}-36840 a_{21}-34440 a_{31} \\
& +120 \sqrt{6}-11840 \sqrt{6} a_{31}+2100000 a_{21} a_{31} \\
& \left.+1330800 a_{31}^{2}+448800 \sqrt{6} a_{31}^{2}+1498800 a_{21}^{2}\right)^{1 / 2} .
\end{aligned}
$$

Also, the global error can be written as

$$
\begin{aligned}
& \left\|\tau_{g}^{(6)}\right\|_{2}=\frac{1}{3600}\left(-15520 a_{21} \sqrt{6}+26400 a_{21}^{2} \sqrt{6}\right. \\
& +600000 a_{21} \sqrt{6} a_{31}-46680 a_{21}-34440 a_{31}+511 \\
& +162 \sqrt{6}-11840 \sqrt{6} a_{31}+2100000 a_{21} a_{31} \\
& \left.+1330800 a_{31}^{2}+448800 \sqrt{6} a_{31}^{2}+2127600 a_{21}^{2}\right) .
\end{aligned}
$$

Now, minimizing the error coefficients in (43) and (44) with respect to the free parameters $a_{21}, a_{31}$, we obtain $a_{21}=$ $4059 / 187793$ and $a_{31}=-1502 / 532215$ which gives $a_{32}=$ $1826 / 569317$. Thus, the error equations for $y, y^{\prime}, y^{\prime \prime}$, and $y^{\prime \prime \prime}$ are computed and given by

$$
\begin{aligned}
\left\|\tau^{(6)}\right\|_{2} & =0, \\
\left\|\tau^{\prime(6)}\right\|_{2} & =8.333333333 \times 10^{-4}, \\
\left\|\tau^{\prime \prime(6)}\right\|_{2} & =1.666666668 \times 10^{-3}, \\
\left\|\tau^{\prime \prime \prime(6)}\right\|_{2} & =1.666666667 \times 10^{-3}
\end{aligned}
$$

and global error norm is

$$
\left\|\tau_{g}^{(6)}\right\|_{2}=2.499999999 \times 10^{-3} .
$$

Therefore, the parameters of the three-stage fifth-order RKFD method denoted by RKFD5 can be represented in Butcher tableau as follows:

$$
\begin{array}{c|ccc}
\frac{3}{5}+\frac{\sqrt{6}}{10} & \frac{4059}{187793} & & \\
\frac{3}{5}-\frac{\sqrt{6}}{10} & -\frac{1502}{532215} & \frac{1826}{569317} & \\
\hline & \frac{19}{1080} & \frac{13}{1080}-\frac{11 \sqrt{6}}{2160} & \frac{13}{1080}+\frac{11 \sqrt{6}}{2160} \\
\frac{1}{18} & \frac{1}{18}-\frac{\sqrt{6}}{48} & \frac{1}{18}+\frac{\sqrt{6}}{48} \\
\frac{1}{9} & \frac{7}{36}-\frac{\sqrt{6}}{18} & \frac{7}{36}+\frac{\sqrt{6}}{18} \\
\frac{1}{9} & \frac{4}{9}-\frac{\sqrt{6}}{36} & \frac{4}{9}+\frac{\sqrt{6}}{36}
\end{array}
$$

\section{Numerical Examples}

In this section, some numerical examples will be solved to show the efficiency of the new RKFD methods of order four 


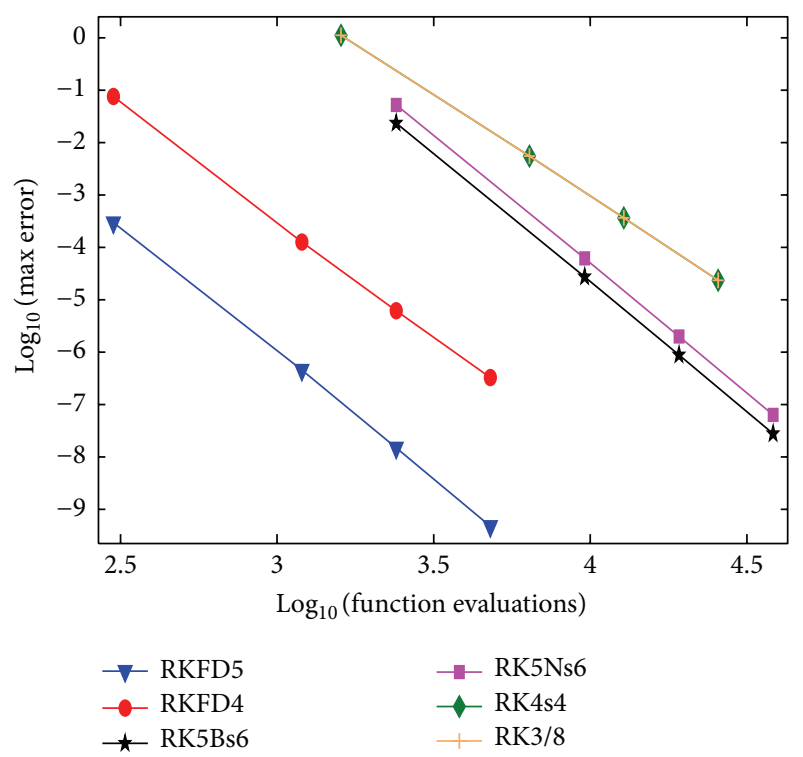

Figure 1: The efficiency curves for Example 1 with $h=0.1 / 2^{i}, i=$ $0,2,3,4$.

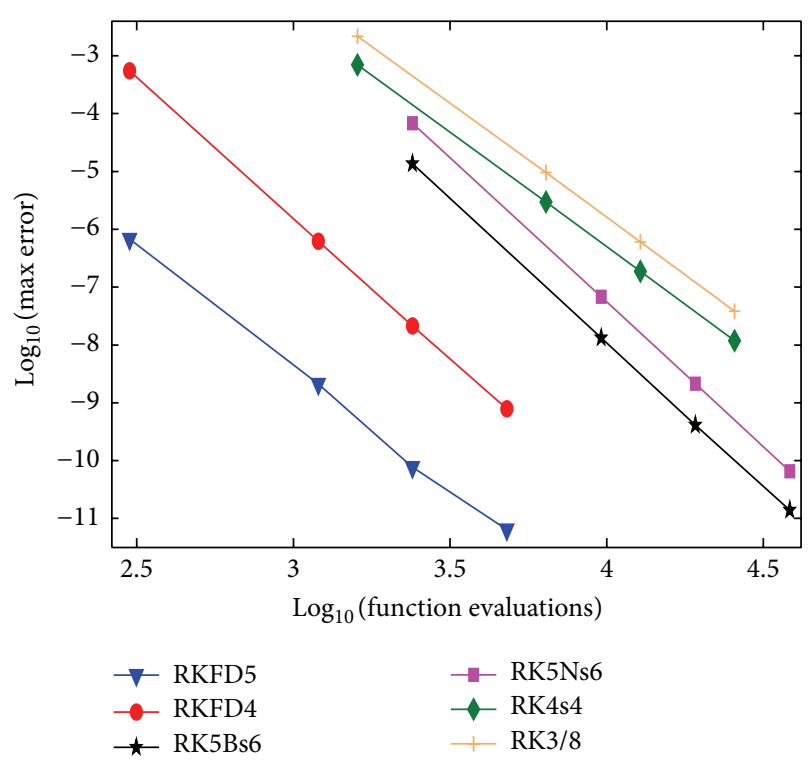

Figure 2: The efficiency curves for Example 2 with $h=0.1 / 2^{i}, i=$ $0,2,3,4$.

and order five, which are denoted by RKFD4 and RKFD5, respectively. The comparison is made with the well known methods in the scientific literature. We use in the numerical comparisons the criteria based on computing the maximum error in the solution $\left(\max\right.$ error $\left.=\max \left(\left|y\left(t_{n}\right)-y_{n}\right|\right)\right)$ which is equal to the maximum between absolute errors of the true solutions and the computed solutions. Figures 1-7 show the efficiency curves of $\log _{10}$ (max error) against the computational effort measured by $\log _{10}$ (function evaluations) required by each method. The following methods are used for comparison:

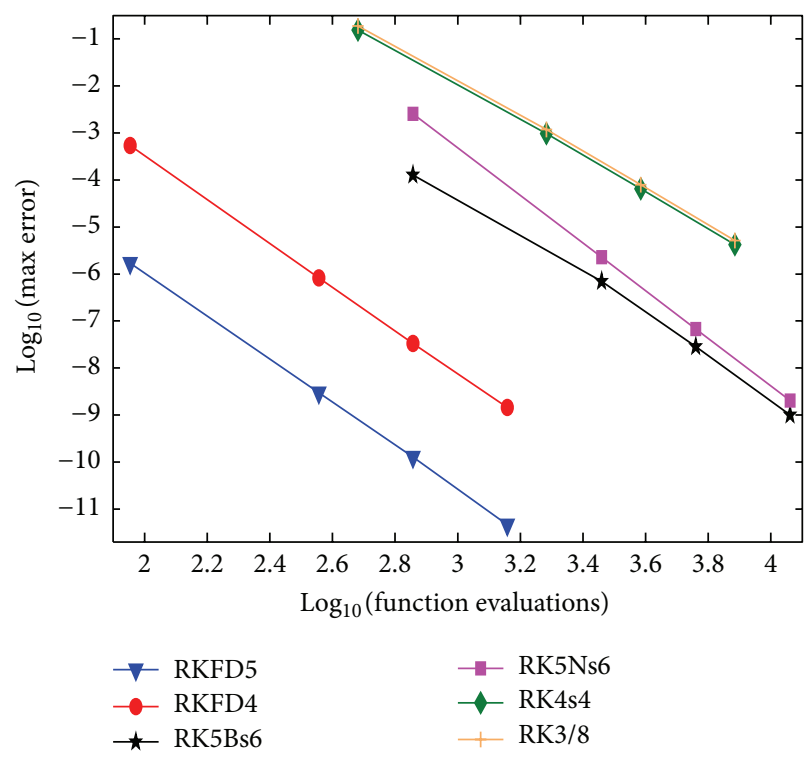

FIGURE 3: The efficiency curves for Example 3 with $h=0.1 / 2^{i}, i=$ $0,2,3,4$.

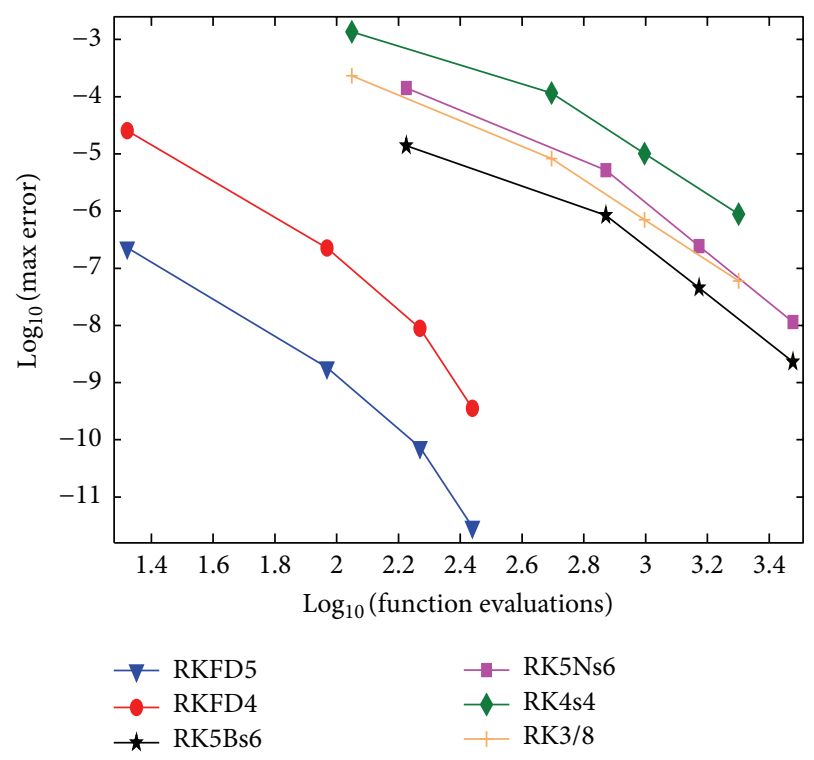

FIgURE 4: The efficiency curves for Example 4 with $h=0.1 / 2^{i}, i=$ $0,2,3,4$

(i) RKFD5: the three-stage fifth-order RKFD method derived in this paper.

(ii) RKFD4: the three-stage fourth-order RKFD method derived in this paper.

(iii) RK5Bs6: the six-stage fifth-order Runge-Kutta method given in Butcher [4].

(iv) RK5Ns6: the six-stage fifth-order Runge-Kutta method given in Hairer [5].

(v) RK4s4: the four-stage fourth-order Runge-Kutta method given in Dormand [14]. 


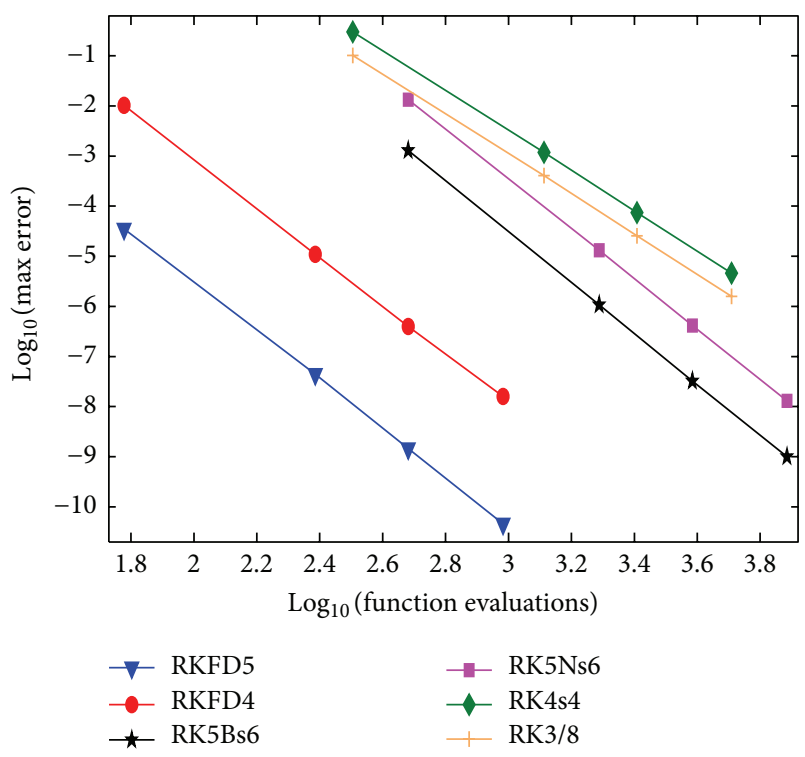

FIgURE 5: The efficiency curves for Example 5 with $h=0.1 / 2^{i}, i=$ $0,2,3,4$.

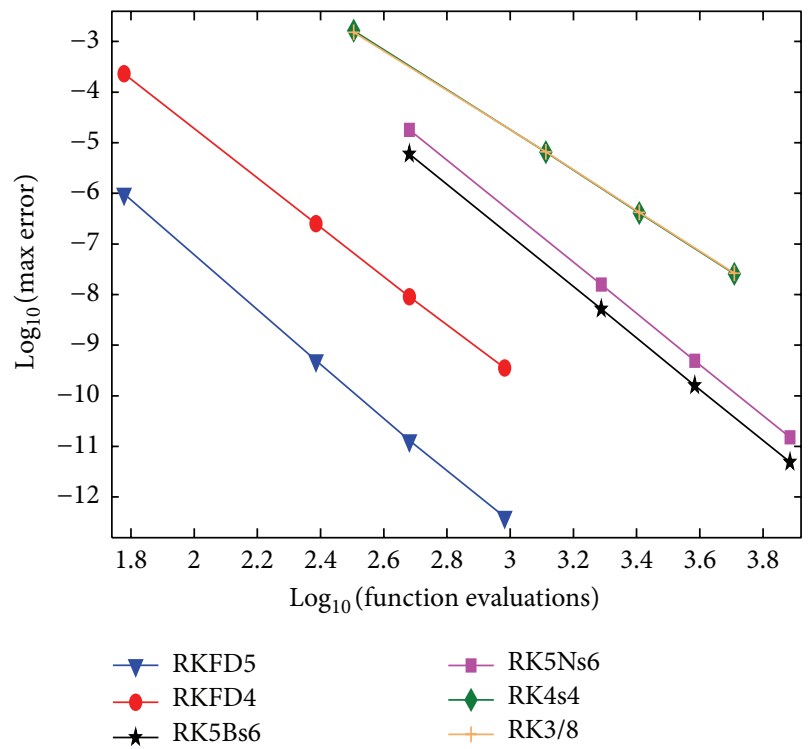

Figure 6: The efficiency curves for Example 6 with $h=0.1 / 2^{i}, i=$ $0,2,3,4$.

(vi) RK3/8: the four-stage fourth-order 3/8 rule RungeKutta method given in Butcher [4].

Example 1. The homogeneous linear problem is as follows:

$$
\begin{aligned}
& y^{(i v)}=-4 y, \\
& \quad y(0)=0, y^{\prime}(0)=1, y^{\prime \prime}(0)=2, y^{\prime \prime \prime}(0)=2 .
\end{aligned}
$$

The exact solution is given by $y(x)=\mathrm{e}^{x} \sin (x)$. The problem is integrated in the interval $[0,10]$.

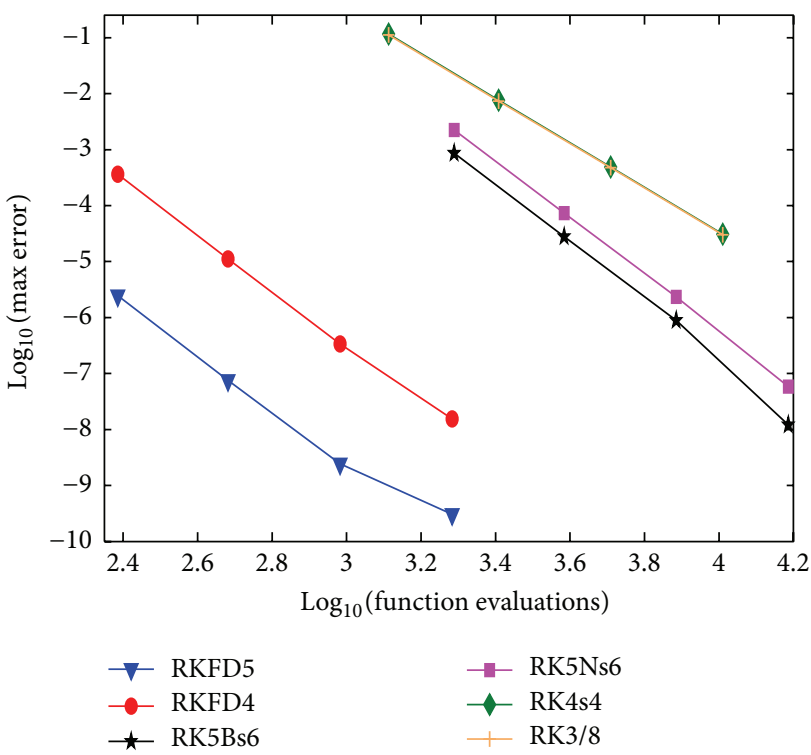

FIgURE 7: The efficiency curves for Example 7 with $h=0.025 / 2^{i}$, $i=0,1,2,3$.

Example 2. The nonhomogeneous nonlinear problem is as follows:

$$
\begin{aligned}
y^{(i v)}= & y^{2}+\cos ^{2}(x)+\sin (x)-1, \\
& y(0)=0, y^{\prime}(0)=1, y^{\prime \prime}(0)=0, y^{\prime \prime \prime}(0)=-1 .
\end{aligned}
$$

The exact solution is given by $y(x)=\sin (x)$. The problem is integrated in the interval $[0,10]$.

Example 3. The homogeneous linear problem with nonconstant coefficients is as follows

$$
\begin{aligned}
y^{(i v)}= & \left(16 x^{4}-48 x^{2}+12\right) y, \\
& y(0)=1, y^{\prime}(0)=0, y^{\prime \prime}(0)=-2, y^{\prime \prime \prime}(0)=0 .
\end{aligned}
$$

The exact solution is given by $y(x)=\mathrm{e}^{-x^{2}}$. The problem is integrated in the interval $[0,3]$.

Example 4. The nonlinear problem is as follows:

$$
\begin{aligned}
y^{(i v)}= & \frac{3 \sin (y)\left(3+2 \sin ^{2}(y)\right)}{\cos ^{7}(y)}, \\
& y(0)=0, y^{\prime}(0)=1, y^{\prime \prime}(0)=0, y^{\prime \prime \prime}(0)=1 .
\end{aligned}
$$

The exact solution is given by $y(x)=\arcsin (x)$. The problem is integrated in the interval $[0, \pi / 4]$. 
Example 5. The linear system is as follows:

$$
\begin{aligned}
& y^{(i v)}=\mathrm{e}^{3 x} u, \\
& y(0)=1, y^{\prime}(0)=-1, y^{\prime \prime}(0)=1, y^{\prime \prime \prime}(0)=-1 . \\
& z^{(i v)}=16 \mathrm{e}^{-x} y, \\
& z(0)=1, z^{\prime}(0)=-2, z^{\prime \prime}(0)=4, z^{\prime \prime \prime}(0)=-8 . \\
& w^{(i v)}=81 \mathrm{e}^{-x} z, \\
& w(0)=1, w^{\prime}(0)=-3, w^{\prime \prime}(0)=9, w^{\prime \prime \prime}(0)=-27 . \\
& u^{(i v)}=256 \mathrm{e}^{-x} w . \\
& u(0)=1, u^{\prime}(0)=-4, u^{\prime \prime}(0)=16, u^{\prime \prime \prime}(0)=-64 .
\end{aligned}
$$

The exact solution is given by

$$
\begin{aligned}
& y=\mathrm{e}^{-x}, \\
& z=\mathrm{e}^{-2 x}, \\
& w=\mathrm{e}^{-3 x}, \\
& u=\mathrm{e}^{-4 x}
\end{aligned}
$$

The problem is integrated in the interval $[0,2]$.

Example 6. The nonlinear system is as follows:

$$
\begin{aligned}
& y^{(i v)}=y+\frac{1}{\sqrt{y^{2}+z^{2}}}-\frac{1}{\sqrt{w^{2}+u^{2}}}, \\
& y(0)=1, y^{\prime}(0)=0, y^{\prime \prime}(0)=-1, y^{\prime \prime \prime}(0)=0 . \\
& z^{(i v)}=z-\frac{1}{\sqrt{y^{2}+z^{2}}}+\frac{1}{\sqrt{w^{2}+u^{2}}}, \\
& z(0)=0, z^{\prime}(0)=1, z^{\prime \prime}(0)=0, z^{\prime \prime \prime}(0)=-1 . \\
& w^{(i v)}=16 w+\frac{1}{\sqrt{y^{2}+z^{2}}}-\frac{1}{\sqrt{w^{2}+u^{2}}}, \\
& w(0)=1, w^{\prime}(0)=0, w^{\prime \prime}(0)=-4, w^{\prime \prime \prime}(0)=0 . \\
& u^{(i v)}=16 u-\frac{1}{\sqrt{y^{2}+z^{2}}}+\frac{1}{\sqrt{w^{2}+u^{2}}} . \\
& u(0)=0, u^{\prime}(0)=2, u^{\prime \prime}(0)=0, u^{\prime \prime \prime}(0)=-8 .
\end{aligned}
$$

The exact solution is given by

$$
\begin{aligned}
& y=\cos (x), \\
& z=\sin (x), \\
& w=\cos (2 x), \\
& u=\sin (2 x) .
\end{aligned}
$$

The problem is integrated in the interval $[0,2]$.
Example 7. The nonlinear system is as follows:

$$
\begin{aligned}
y^{(i v)}= & \frac{z^{2}}{w}, \\
& y(0)=1, y^{\prime}(0)=1, y^{\prime \prime}(0)=1, y^{\prime \prime \prime}(0)=1 . \\
z^{(i v)}= & 16 \frac{w^{2}}{u}, \\
& z(0)=1, z^{\prime}(0)=2, z^{\prime \prime}(0)=4, z^{\prime \prime \prime}(0)=8 . \\
w^{(i v)}= & 81 \frac{u^{2}}{y^{5}}, \\
w & (0)=1, w^{\prime}(0)=3, w^{\prime \prime}(0)=9, w^{\prime \prime \prime}(0)=27 . \\
u^{(i v)}= & 256 y^{4} . \\
& u(0)=1, u^{\prime}(0)=4, u^{\prime \prime}(0)=16, u^{\prime \prime \prime}(0)=64 .
\end{aligned}
$$

The exact solution is given by

$$
\begin{aligned}
& y=\mathrm{e}^{x}, \\
& z=\mathrm{e}^{2 x}, \\
& w=\mathrm{e}^{3 x}, \\
& u=\mathrm{e}^{4 x} .
\end{aligned}
$$

The problem is integrated in the interval $[0,2]$.

\section{Conclusion}

This paper deals with Runge-Kutta type method denoted by RKFD method for directly solving special fourth-order ODEs of the form $y^{(i v)}(x)=f(x, y)$. First, we derived the order conditions for RKFD method, which were then used to construct three-stage fourth- and fifth-order RKFD methods. The methods are denoted by RKFD5 and RKFD4, respectively. We also proved that the RKFD method is zero-stable. From the numerical results, we observed that the new RKFD methods are more competent as compared with the existing Runge-Kutta methods in the scientific literature. From the numerical results, we conclude that the new RKFD methods are computationally more efficient in solving special fourthorder ODEs and outperformed the existing methods in terms of error precision and number of function evaluations.

\section{Conflict of Interests}

The authors declare that there is no conflict of interests regarding the publication of this paper.

\section{References}

[1] P. Onumanyi, U. W. Sirisena, and S. N. Jator, "Continuous finite difference approximations for solving differential equations," International Journal of Computer Mathematics, vol. 72, no. 1, pp. 15-27, 1999. 
[2] D. Sarafyan, "New algorithms for the continuous approximate solution of ordinary differential equations and the upgrading of the order of the processes," Computers \& Mathematics with Applications, vol. 20, no. 1, pp. 77-100, 1990.

[3] G. Dahlquist, "On accuracy and unconditional stability of linear multistep methods for second order differential equations," BIT Numerical Mathematics, vol. 18, no. 2, pp. 133-136, 1978.

[4] J. C. Butcher, Numerical Methods for Ordinary Differential Equations, John Wiley \& Sons, New York, NY, USA, 2nd edition, 2008.

[5] E. Hairer, S. P. Nørsett, and G. Wanner, Solving Ordinary Differential Equations I: Nonstiff Problems, vol. 8 of Springer Series in Computational Mathematics, Springer, Berlin, Germany, 2nd edition, 1993.

[6] S. N. Jator and J. Li, "A self-starting linear multistep method for a direct solution of the general second-order initial value problem," International Journal of Computer Mathematics, vol. 86, no. 5, pp. 827-836, 2009.

[7] D. O. Awoyemi, "A new sixth-order algorithm for general second order ordinary differential equation," International Journal of Computer Mathematics, vol. 77, no. 1, pp. 117-124, 2001.

[8] S. J. Kayode, "An efficient zero-stable numerical method for fourth-order differential equations," International Journal of Mathematics and Mathematical Sciences, vol. 2008, Article ID 364021, 10 pages, 2008.

[9] Z. A. Majid and M. B. Suleiman, "Direct integration implicit variable steps method for solving higher order systems of ordinary differential equations directly," Sains Malaysiana, vol. 35, no. 2, pp. 63-68, 2006.

[10] M.-K. Jain, S. R. K. Iyengar, and J. S. V. Saldanha, "Numerical solution of a fourth-order ordinary differential equation," Journal of Engineering Mathematics, vol. 11, no. 4, pp. 373-380, 1977.

[11] N. Waeleh, Z. A. Majid, and F. Ismail, "A new algorithm for solving higher order IVPs of ODEs," Applied Mathematical Sciences, vol. 5, no. 53-56, pp. 2795-2805, 2011.

[12] D. O. Awoyemi and O. M. Idowu, "A class of hybrid collocation methods for third-order ordinary differential equations," International Journal of Computer Mathematics, vol. 82, no. 10, pp. 1287-1293, 2005.

[13] S. N. Jator, "Solving second order initial value problems by a hybrid multistep method without predictors," Applied Mathematics and Computation, vol. 217, no. 8, pp. 4036-4046, 2010.

[14] J. R. Dormand, Numerical Methods for Differential Equations. A Computational Approach, Library of Engineering Mathematics, CRC Press, Boca Raton, Fla, USA, 1996.

[15] W. Gander and D. Gruntz, "Derivation of numerical methods using computer algebra," SIAM Review, vol. 41, no. 3, pp. 577$593,1999$.

[16] J. D. Lambert, Numerical Methods for Ordinary Differential Systems, The Initial Value Problem, John Wiley \& Sons, London, UK, 1991.

[17] P. Henrici, Elements of Numerical Analysis, John Wiley \& Sons, New York, NY, USA, 1964.

[18] J. R. Dormand, M. E. A. EL-Mikkawy, and P. J. Prince, "Families of Runge-Kutta Nystrom formulae," IMA Journal of Numerical Analysis, vol. 7, pp. 235-250, 1987. 


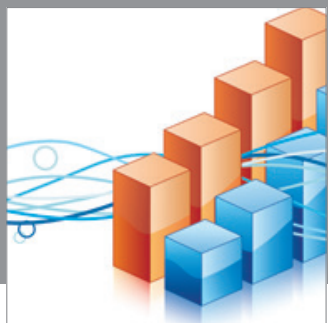

Advances in

Operations Research

mansans

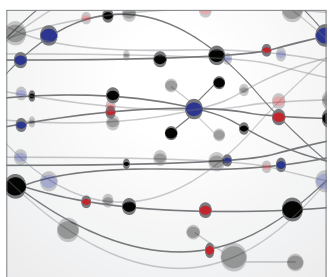

The Scientific World Journal
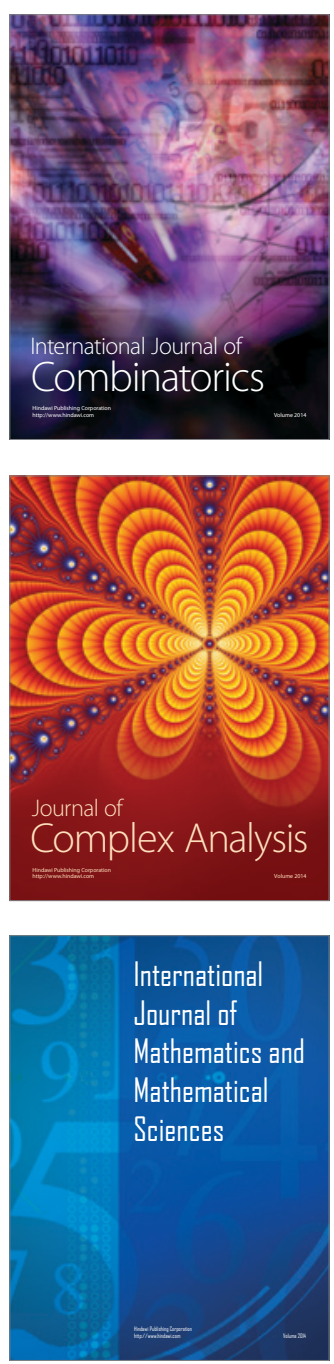
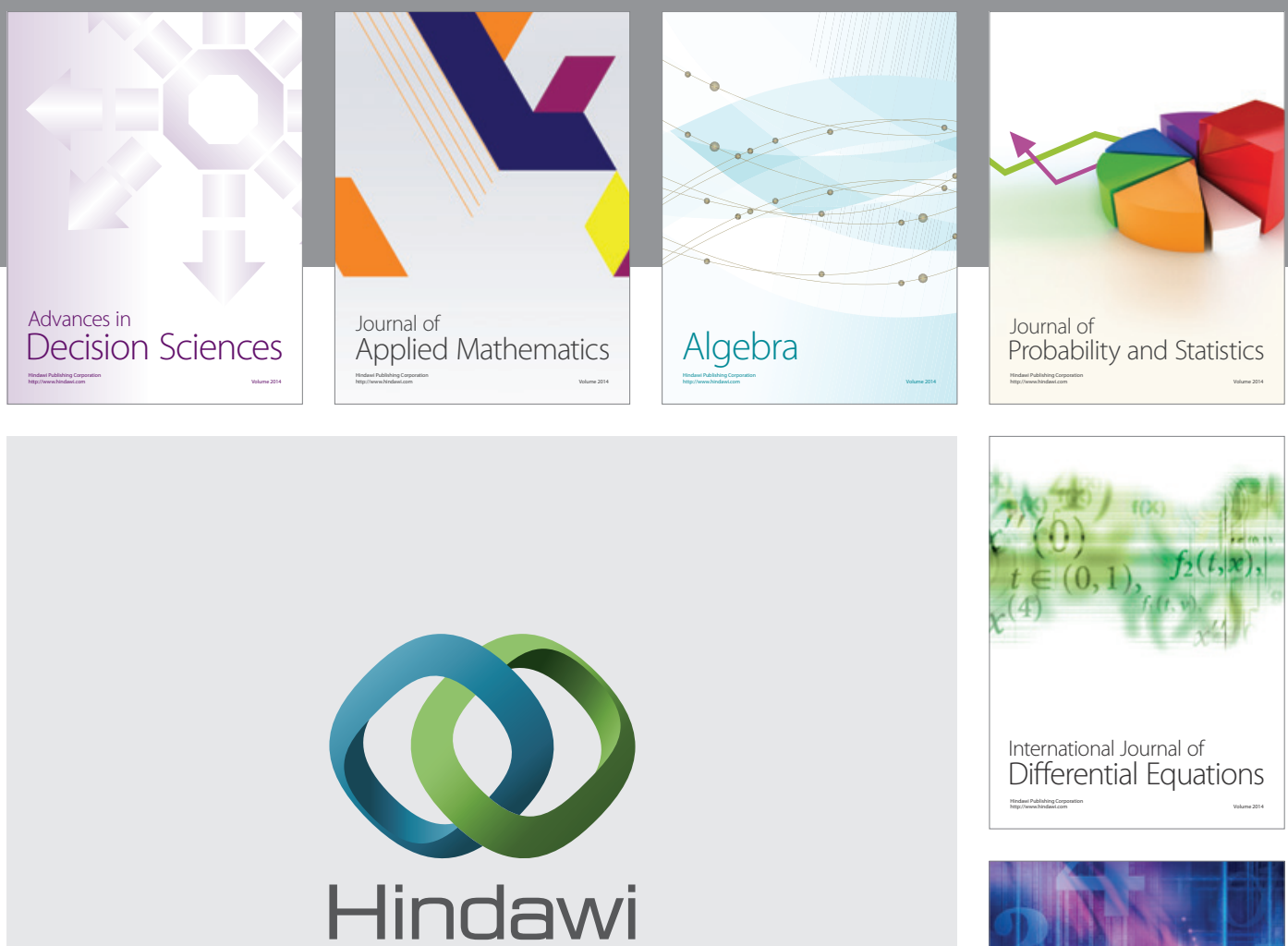

Submit your manuscripts at http://www.hindawi.com
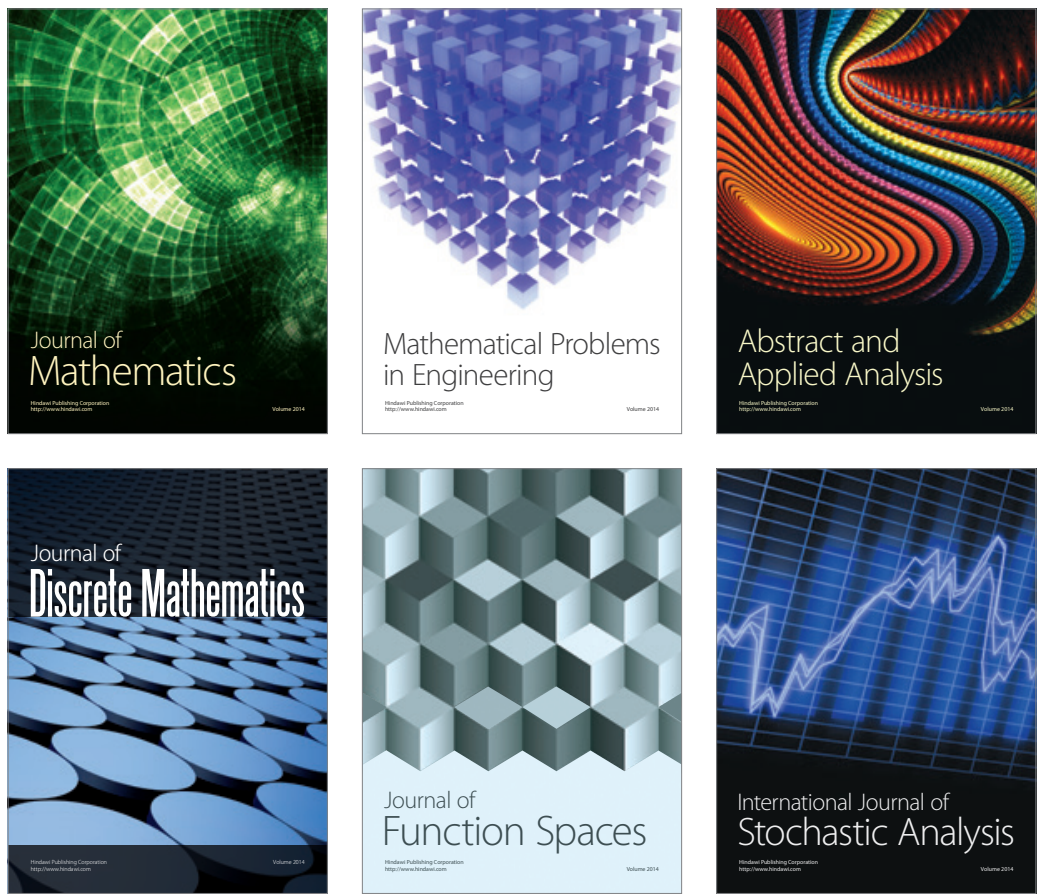

Journal of

Function Spaces

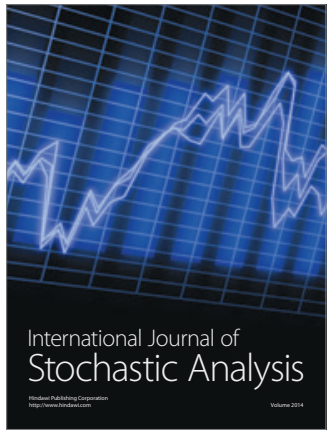

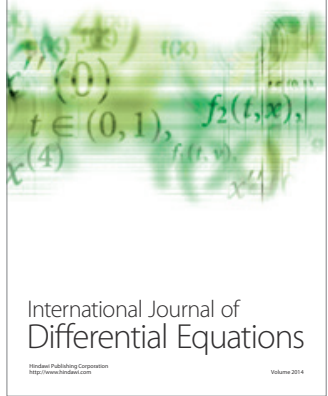
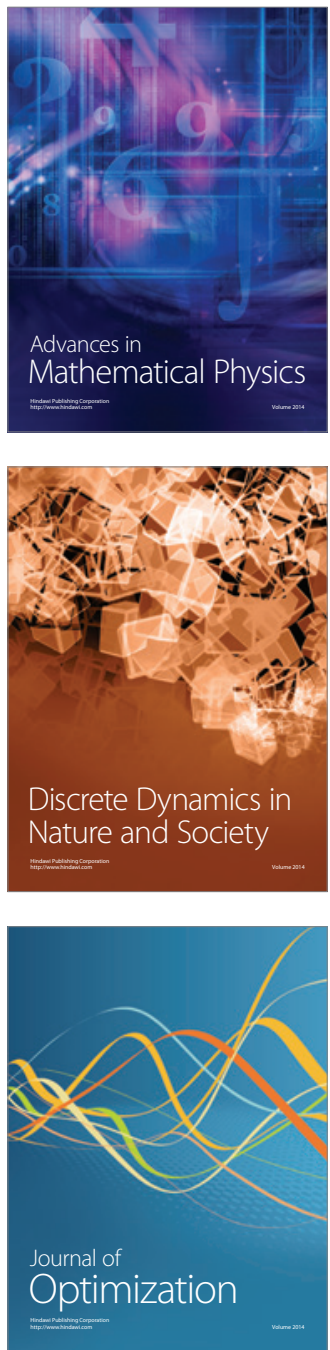